J. Clin. Chem. Clin. Biochem.

Vol. 19, 1981, pp. 347-350

\title{
Clinical Evaluation of Two Calculation Methods for a Free Thyroxine Assay
}

\author{
By A. J. Bakker and Ina Terpstra \\ Clinical Chemistry Laboratory (Director: Dr. P. C. van der Schaaf) Leeuwarden, The Netherlands
}

(Received June 12/December 1, 1980)

Summary: A commercial assay for the measurement of the free thyroxine concentration in serum has been subjected to a change of method of calculation, together with a change of antibody. The clinical significance of both methods of calculation, before and after the change of antibody, has been investigated.

Before the change of antibody the results of the original method of calculation were in slightly better agreement with the clinical data. After the change of antibody the results of the modified method of calculation were in better agreement with the clinical data.

\section{Klinische Bewertung von zwei Berechnungsmethoden für eine Bestimmung des freien Thyroxins}

Zusammenfassung: Eine käufliche Bestimmungsmethode für freies Thyroxin im Serum wurde hinsichtlich des Berechnungsmodus sowie des verwendeten Antikörpers modifiziert. Die klinische Bedeutung beider Berechnungsmethoden vor und nach Wechsel des Antikörpers wurde geprüft.

Vor dem Wechsel des Antikörpers stimmten die Ergebnisse der Originalmethode der Berechnung geringfügig besser mit den klinischen Daten überein. Nach dem Wechsel des Antikörpers stimmten die Ergebnisse der modifizierten Berechnungsmethode besser mit den klinischen Daten überein.

\section{Introduction}

The amount of free thyroxine $\left(\mathrm{T}_{4}\right)$ in serum is usually determined by cumbersome methods involving timeconsuming equilibrium dialysis (1-4), so these methods are not in common use for the routine evaluation of thyroid function.

The recently introduced commercial kits for free $\mathrm{T}_{4}$ determination lack these disadvantages, so they can be used for routine purposes.

One of these commercial kits for free $\mathrm{T}_{4}$ determination has been subjected to a change of antibody as well as to a change of method of calculation. We have been evaluating the clinical consequences of both methods of calculation before and after the change of antibody.

\section{Materials and Methods}

\section{Samples}

All sera were stored at $-20^{\circ} \mathrm{C}$ prior to assay. Specimens were thawed and allowed to reach room temperature before analysis.
Procedures

The free $T_{4}$-immophase kinetic assay, which was used, was obtained from Corning Medical (Medfield, U.S.A.).

This is a two-tube assay. An amount of radiolabelled $\mathrm{T}_{4}$ is added to the sample, and after a period of equilibration, a solid phase antibody is added and incubated for thirty minutes. The radioactivity bound to the solid phase antibody is measured. In the $A$ series tube the rate of binding measured depends on the amount of free $T_{4}$.

In the $B$ series tube the rate of binding measured depends on the amount of total $\mathrm{T}_{4}$, because previous added thimerosal liberates $\mathrm{T}_{4}$ from its binding proteins.

In the original method of calculation the socalled relative rate \%

$$
\text { (= } \left.=\frac{\text { ppm bound tube } A}{\text { cpm bound tube } B} \times 100 \%\right)
$$

is a function of free $\mathrm{T}_{4}$.

In the modified method of calculation, calculation of free $\mathrm{T}_{4}$ depends on the prior calculation of total $T_{4}$. Total $T_{4}$ can be calculated because the fraction bound in the $B$ series

$$
\text { (= } \frac{\text { cpm bound tube } B}{\text { total counts } B} \text { ) }
$$

is a function of total $\mathrm{T}_{4}$.

Free $T_{4}$ can then be calculated because the product of total $T_{4}$ and the fraction bound in the $A$ series

$$
\text { ( }=\frac{\text { cpm bound tube } A}{\text { total counts }} \text { ) }
$$

is a function of free $T_{4}$. 
Before the antibody was changed serum samples from 88 blood donors and 145 unselected patients were examined. After the change of antibody, serum samples from 155 blood donors, 225 unselected patients and 95 pregnant euthyro id women were evaluated. The "assay data" of both methods of calculation before and after the change of antibody were compared by using the least-squares method (5).

\section{Results}

\section{Precision}

The day-to-day variability of the assay for free $T_{4}$ both before and after the change of antibody has been considered. The influence of the method of calculation on the day-to-day variability of the assay for free $T_{4}$ has also been evaluated. The results shown in table 1 were obtained in the routine use of this assay for free $T_{4}$.

Tab. 1. Influence of the method of calculation on the day-to-day variability of the assay for free $T_{4}$ before and after a change of antibody. The mean $(\bar{x})$ and the standard deviation (s) are expressed in $\mathrm{pmol} / \mathrm{l}$.

$\begin{array}{ll}\mathrm{FT}_{4} \mathrm{OC} & =\text { original method of calculation for free } \mathrm{T}_{4} . \\ \mathrm{FT}_{4} \mathrm{nc} & =\text { modified method of calculation for free } \mathrm{T}_{4} . \\ \text { Ref. } 1 & =\text { reference control serum 1, supplied by Corning } \\ & \text { medical, Medfield, U.S.A. }\end{array}$

NMS $1 / \mathrm{II}^{\mathrm{A}}=$ control sera 1 and IIA, supplied by Nuclear Medical Systems Inc., Newport Beach, U.S.A.

\begin{tabular}{|c|c|c|c|c|c|c|}
\hline \multicolumn{4}{|c|}{ Method of } & \multicolumn{3}{|c|}{ Free thýroxine } \\
\hline $\begin{array}{l}\text { Anti- } \\
\text { body }\end{array}$ & $\begin{array}{l}\text { calcula- } \\
\text { tion }\end{array}$ & Control & $\mathrm{N}$ & $\begin{array}{l}\overline{\mathrm{x}} \\
(\mathrm{pmol} / \mathrm{l})\end{array}$ & $\begin{array}{l}s \\
(\mathrm{pmol} / \mathrm{l})\end{array}$ & $\begin{array}{l}\text { CV } \\
(\%)\end{array}$ \\
\hline \multirow[t]{2}{*}{ Original } & $\mathrm{FT}_{4} \mathrm{OC}$ & $\begin{array}{l}\text { Ref. } 1 \\
\text { NMS IIA }\end{array}$ & $\begin{array}{l}22 \\
22\end{array}$ & $\begin{array}{l}19.05 \\
37.07\end{array}$ & $\begin{array}{l}1.03 \\
2.19\end{array}$ & $\begin{array}{l}5.4 \\
5.9\end{array}$ \\
\hline & $\mathrm{FT}_{4} \mathrm{nc}$ & $\begin{array}{l}\text { Ref. } 1 \\
\text { NMS IIA }\end{array}$ & $\begin{array}{l}22 \\
22\end{array}$ & $\begin{array}{l}22.14 \\
42.34\end{array}$ & $\begin{array}{l}1.16 \\
2.44\end{array}$ & $\begin{array}{l}5.2 \\
5.8\end{array}$ \\
\hline \multirow[t]{2}{*}{ Changed } & $\mathrm{FT}_{4} \mathrm{OC}$ & $\begin{array}{l}\text { Ref. } 1 \\
\text { NMS } 1\end{array}$ & $\begin{array}{l}23 \\
23\end{array}$ & $\begin{array}{l}19.18 \\
38.48\end{array}$ & $\begin{array}{l}1.29 \\
5.02\end{array}$ & $\begin{array}{r}6.7 \\
13.0\end{array}$ \\
\hline & $\mathrm{FT}_{4} \mathrm{nc}$ & $\begin{array}{l}\text { Ref. } 1 \\
\text { NMS } 1\end{array}$ & $\begin{array}{l}23 \\
23\end{array}$ & $\begin{array}{l}20.59 \\
32.43\end{array}$ & $\begin{array}{l}0.90 \\
2.70\end{array}$ & $\begin{array}{l}4.4 \\
8.3\end{array}$ \\
\hline
\end{tabular}

Comparison of the "assay data" of both methods of calculation for free $T_{4}$

Before the change of antibody, comparison of the values for free $\mathrm{T}_{4}$ by both methods of calculation showed a good correlation, as shown in figure 1.

After the change of antibody, the values for free $T_{4}$ by both methods showed a bad correlation. This is shown in figure 2 .

\section{Reference values}

In order to establish the clinical significance of the changes, it is necessary to obtain reference values for the free $T_{4}$ for both methods of calculation, both before and after the change of antibody.

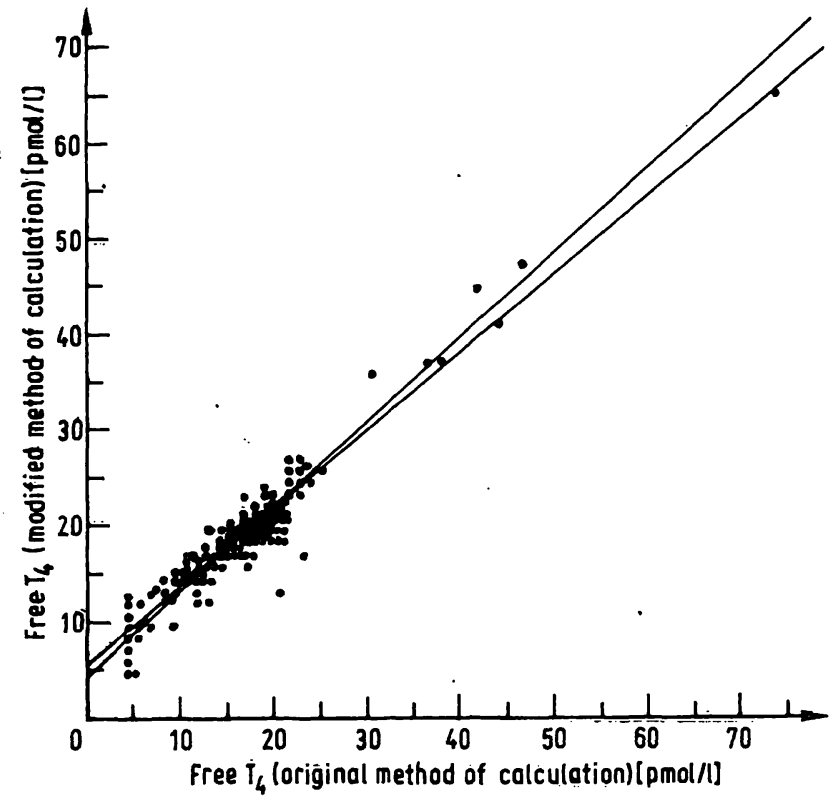

Fig. 1. Comparison of two methods of calculation for free $T_{4}$ before the change of antibody.

$\mathrm{FT}_{4} \mathrm{OC}=$ original method of calculation;

$\mathrm{FT}_{4} \mathrm{nc}=$ modified method of calculation;

$\mathrm{FT}_{4} \mathrm{OC}=1.141 \cdot \mathrm{FT}_{4} \mathrm{nc}-5.019$

$\mathrm{FT}_{4} \mathrm{nc}=0.809 \cdot \mathrm{FT}_{4} \mathrm{OC}+5.633 ; \mathrm{N}=145 ; \mathrm{r}=0.961$.

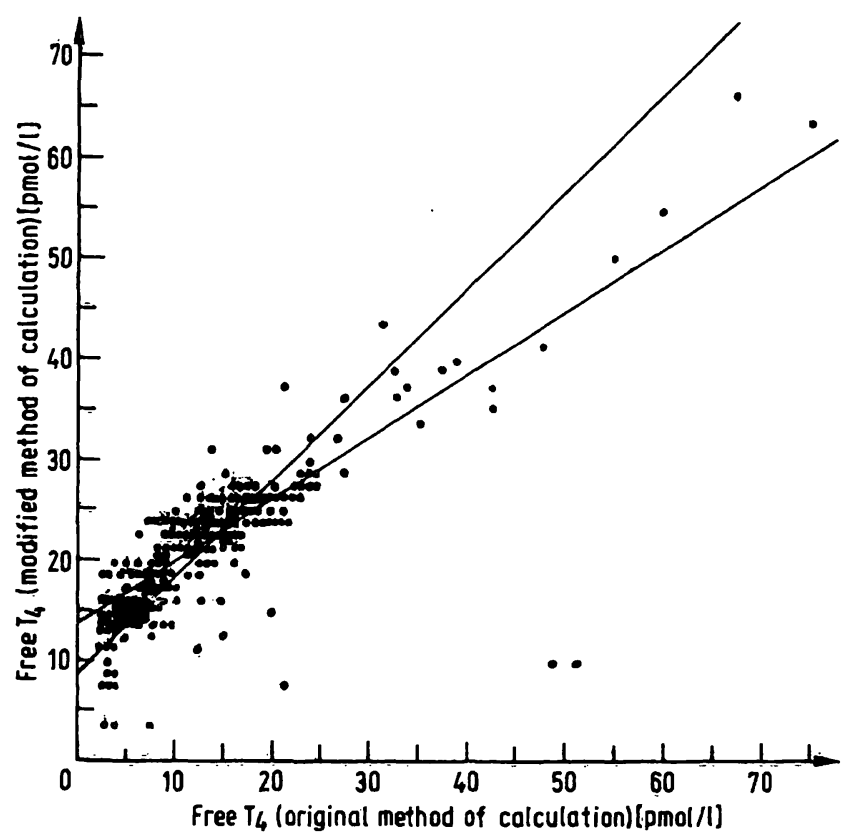

Fig. 2. Comparison of two methods of calculation for free $T_{4}$ after the change of antibody.

$\mathrm{FT}_{4} \mathrm{OC}=$ original method of calculation;

$\mathrm{FT}_{4} \mathrm{nc}=$ modified method of calculation;

$\mathrm{FT}_{4} \mathrm{OC}=1.046 \cdot \mathrm{FT}_{4} \mathrm{nc}-9.212$

$\mathrm{FT}_{4} \mathrm{nc}=0.615 \cdot \mathrm{FT}_{4} \mathrm{Oc}+13.68 .6 ; \mathrm{N}=225 ; \mathrm{r}=0.802$.

These reference values were calculated both for a group of blood donors and a group of selected euthyroid. patients, who fulfil the following criteria:

- they have never been treated for any kind of thyroid dysfunction. 
- they are not using any drug known to influence thyroid function, concentration of $\mathrm{T}_{4}$-binding proteins or interaction between $\mathrm{T}_{4}$ and its binding proteins.

The $95 \%$ interval for both control groups and the reference values based on these two populations are shown in table 2.

Tab. 2. The $95 \%$ interval for free $\mathrm{T}_{4}$ (in pmol/1) in two euthyroid control groups and the reference values based on both groups. Free $T_{4}$ is calculated by both methods of calculation ( $\mathrm{FT}_{4} \mathrm{Oc}=$ original method of calculation and $\mathrm{FT}_{\mathbf{4}} \mathrm{nc}=$ modified method of calculation), before and after the change of antibody.

\begin{tabular}{|c|c|c|c|c|}
\hline \multirow[b]{2}{*}{$\begin{array}{l}\text { Anti- } \\
\text { body }\end{array}$} & \multirow[b]{2}{*}{$\begin{array}{l}\text { Method } \\
\text { of calcu- } \\
\text { lation }\end{array}$} & \multicolumn{2}{|c|}{ Free thyroxine } & \multirow[b]{2}{*}{$\begin{array}{l}\text { Reference } \\
\text { values }\end{array}$} \\
\hline & & $\begin{array}{l}\text { Blood } \\
\text { donors }\end{array}$ & $\begin{array}{l}\text { Selected } \\
\text { euthyroid } \\
\text { patients }\end{array}$ & \\
\hline \multirow[t]{2}{*}{ Original } & $\mathrm{FT}_{4} \mathrm{OC}$ & $\begin{array}{l}10.3-23.1 \\
(\mathrm{~N}=88)\end{array}$ & $\begin{array}{l}10.3-25.7 \\
(\mathrm{~N}=67)\end{array}$ & $10.3-24.4$ \\
\hline & $\mathrm{FT}_{4} \mathrm{nc}$ & $\begin{array}{l}15.4-23.1 \\
(\mathrm{~N}=88)\end{array}$ & $\begin{array}{l}15.4-27.0 \\
(\mathrm{~N}=67)\end{array}$ & $15.4-25.7$ \\
\hline \multirow[t]{2}{*}{ Changed } & $\mathrm{FT}_{4} \mathrm{OC}$ & $\begin{array}{c}7.4-22.4 \\
(\mathrm{~N}=157)\end{array}$ & $\begin{array}{l}5.2-21.9 \\
(\mathrm{~N}=72)\end{array}$ & $\left.7.4-22.4^{*}\right)$ \\
\hline & $\mathrm{FT}_{4} \mathrm{nc}$ & $\begin{array}{l}16.2-28.6 \\
(\mathrm{~N}=157)\end{array}$ & $\begin{array}{l}15.4-29.9 \\
(\mathrm{~N}=72)\end{array}$ & $15.4-28.6$ \\
\hline
\end{tabular}

*) This reference value is based on the blood donors only; otherwise the lower limit would be lower than the lowest standard.

\section{Clinical significance of the "assay data" of both methods of calculation for free $T_{4}$}

The patients were divided on clinical criteria into one of the four following groups:

1. Clinically euthyroid. This group included patients using drugs known to influence thyroid function and the concentration of $\mathrm{T}_{4}$-binding proteins.

2. Clinically hyperthyroid.

3. Clinically hypothyroid.

4. Hypothyroid patients who became euthyroid after adequate substitution therapy.

In all these patients thyrotropin levels had returned to normal, which was considered as biochemical evidence for adequate substitution therapy.

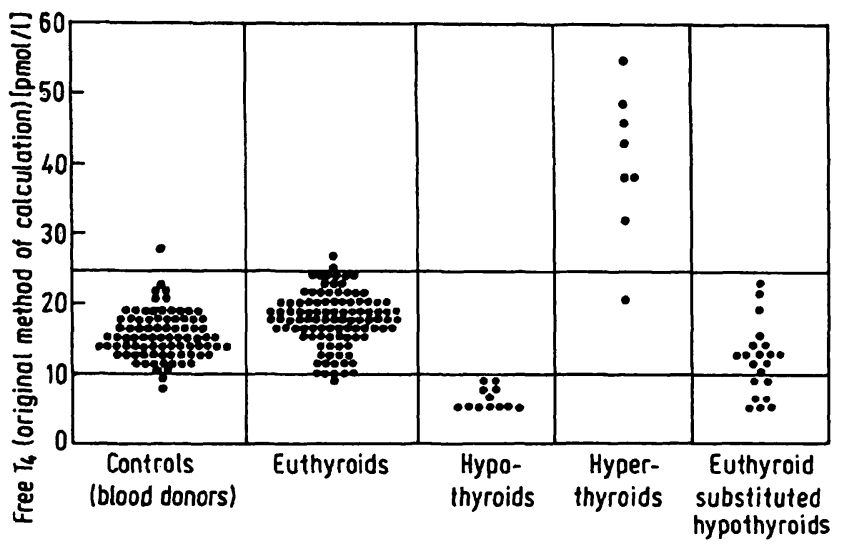

Fig. 3. Distribution of free $T_{4}$-values, calculated by the original method, before the change of antibody.

( $-=$ reference values)

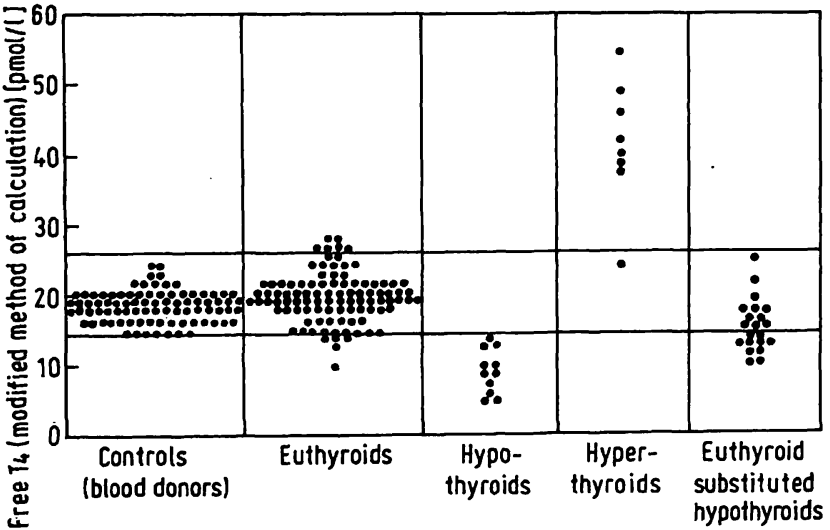

Fig. 4. Distribution of free $T_{4}$-values, calculated by the modified method, before the change of antibody.

(- = reference values)

The two methods of calculation were compared for these four groups and a group of blood donors. The distribution of free $\mathrm{T}_{4}$-values before the change of antibody are shown in figure 3 and 4 . After the change of antibody, a group of euthyroid pregnant women was also included in the comparison of both methods of calculation. The distribution of both free $T_{4}$-values are shown in figure 5 and 6 . The numerical agreement between clinical data and "assay data" is shown in table 3.

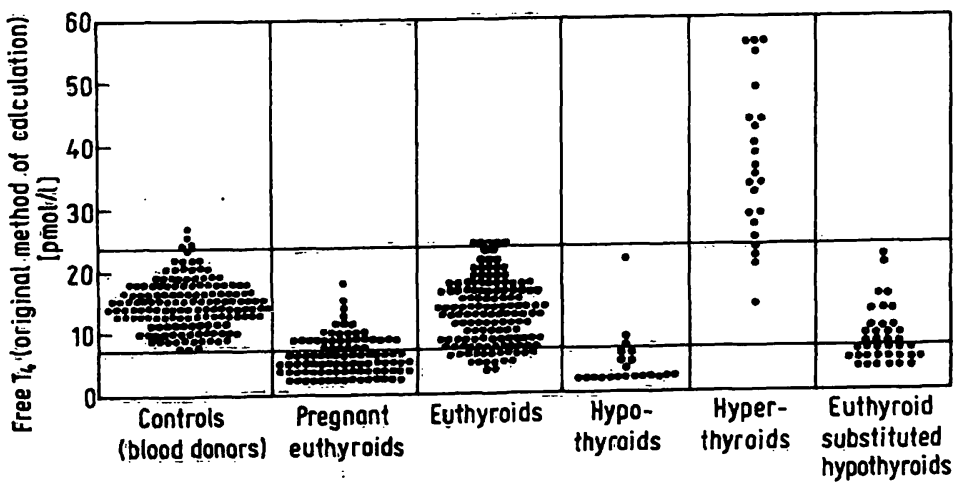

Fig. 5. Distribution of free $T_{4}$-values, calculated by the original method, after the change of antibody.

( $-=$ reference values) 
Tab. 3. Numerical agreement of clinical data and the "assay data" of free $T_{4}$ calculated with two methods of calculation, before and after the change of antibody. $\left(\mathrm{FT}_{4} \mathrm{OC}=\right.$ original method of calculation; $\mathrm{FT}_{4} \mathrm{nc}=$ modified method of calculation; $\mathrm{N}=$ number of patients).

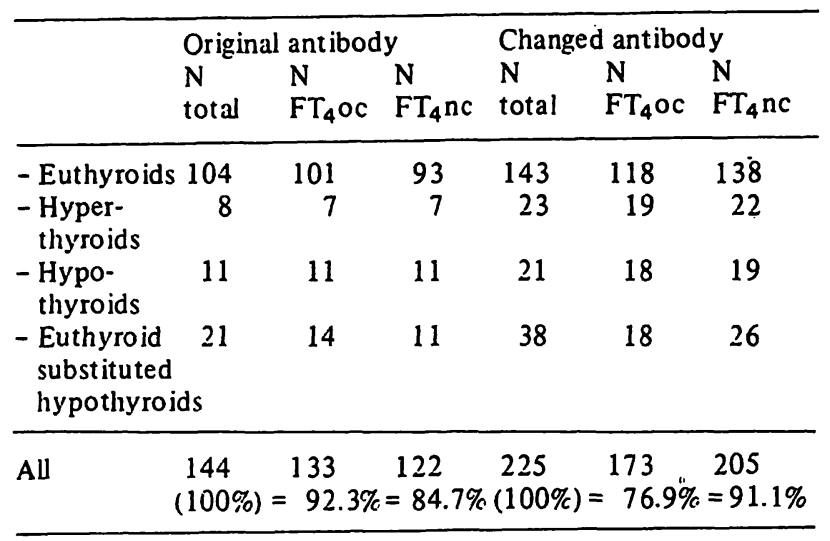

\section{Discussion}

Recently the method of calculation for this assay for free $T_{4}$ was discussed elsewhere $(6,7)$. As was pointed out on theoretical grounds, the modified method of calculation for free $T_{4}$ should be preferred. Our results support this conclusion. In the first group of patients, before the change of antibody, the results did not differ much, but no euthyroid pregnant women could be included in this study because of the change of antibody. In the second group of patients, after the change of antibody, the modified method of calculation for free $\mathrm{T}_{4}$ was, however, superior. This group of patients included euthyroid pregnant women. In contrast with the results of others $(8,9)$ our results with the Corning free $\mathrm{T}_{4}$ kit are not influenced by abnormally elevated concentrations of $\mathrm{T}_{4}$-binding proteins. This is illustrated by the free $\mathrm{T}_{4}$ results, obtained by analysis of samples of the euthyroid pregnant women (fig. 6). This difference was probably caused by the changing of antibody.

\section{Acknowledgements}

We would like to thank the following physicians, Dr. A. Bosma, Dr. J. F. Feddes, Dr. S. S. Hofstra, Dr. J. Houwerzijl, Dr. M. P. Leemhuis and Dr.J.P. Samson for providing the clinical data of their patients.

Furthermore we thank Miss L.J. Oppenheimer and Mr. J. Kattestaart for their technical assistance and Mrs. R. G. Bakker-van der Schaaf for her indispensible editorial assistance.

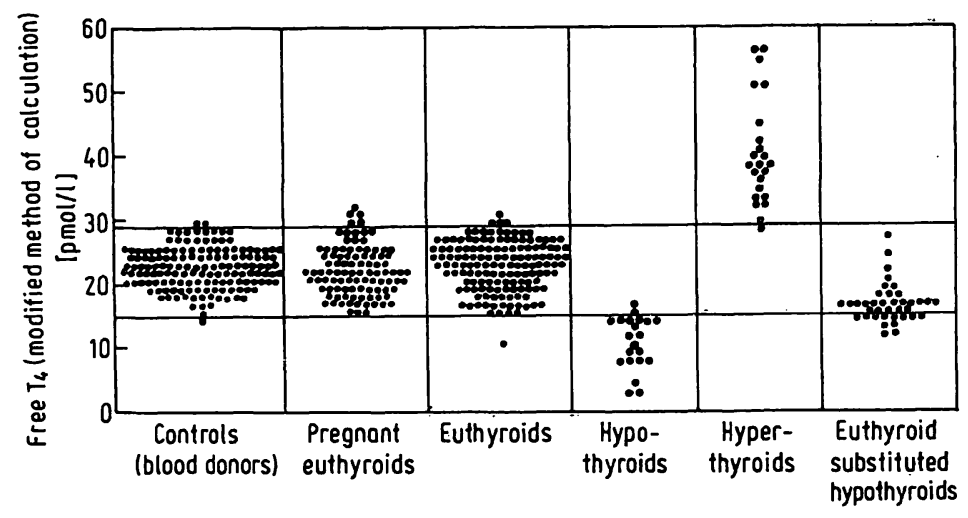

Fig. 6. Distribution of free $T_{4}$-values, calculated by the modified method, after the change of antibody.

$(-=$ reference values $)$

\section{References}

1. Fang, V. S. \& Selenkow, H. A. (1972), Clin. Chem. 16 $185-190$.

2. McDonald L. J., Robin, N. I. \& Siegel, L. (1978), Clin. Chem. $24,652-656$

3. Jiang, N. S. \& Tue, K. A. (1977), Clin. Chem. 23, 1679-1683

4. Petersen, B. A., Giese, R. W., Larsen, P. R. \& Kargel, 'B. L. (1977), Clin. Chem. 23, 1389-1396.

5. Sachs, L. (1968), Statistische Auswertungsmethoden, 374-382, Springer-Verlag, Berlin.
6. Ekins R. (1979), Lancet $I, 1190-1191$.

7. Fullarton, J. \& Lidgard, G. P. (1979), Lancet II, 51.

8. Braverman, L. E., Abreau, C. M., Brock, P., Kleinmann, R., Fournier, L., Odstrchel, G. \& Schoemaker, H. J. P. (1980), J. Nucl. Med. 21, 529-539.

9. Witherspoon, L. R., Shuler, S. E., Garcia, M. M. \& Zollinger, L. A. (1980), J. Nucl. Med. 21, 529-539.

Drs. A. J. Bakker Clinical Chemistry Laboratory Jelsumerstraat 6 NL-8917 EN Leeuwarden 
J. Clin. Chem. Clin. Biochem.

Vol. 19, 1981, pp. 351-356

\title{
Ein einfaches Modell zur Haltbarkeitsberechnung
}

\author{
Von P. Schläpfer und F. Hänni \\ F. Hoffmann-La Roche \& Co. AG, Basel \\ (Eingegangen am 18. März 1980/9. Februar 1981)
}

Zusammenfassung: Mit Hilfe eines einfachen Modells wird die Haltbarkeitsberechnung unter Berücksichtigung der Geschwindigkeitskonstanten und deren Streuung sowie des Gesamtanalysenfehlers bei gegebener Verlusttoleranz und Ausschußwahrscheinlichkeit aufgezeigt. An den Beispielen Kreatinkinase und saure Phosphatase als kritischen Komponenten einer Kontrollprobe wird das Verfahren näher erläutert.

\section{A simple model for the calculation of stability}

Summary: A simple model for stability calculation is demonstrated, taking into consideration the velocity constant, its variance, the analytical error by given loss tolerance and the rejection probability. The procedure is explained in detail and exemplified by creatine kinase and acid phosphatase, both relative instable components in controi samples.

\section{Einleitung}

Bei der Entwicklung und Kommerzialisierung von biochemischen Produkten ist die Stabilitätsprüfung ein wichtiger Bestandteil der Qualitätssicherung. Das Spektrum der Haltbarkeitsprüfung umfaßt Streßversuche (durch thermische Belastung) (1), um kurzfristig zu einer verbindlichen Aussage zu kommen, und die Langzeitprïfung.

In Zusammenhang mit der Entwicklung von Kontrollproben zur Qualitätskontrolle im klinisch-chemischen Labor wurde nach einem allgemein anwendbaren Modell zur Haltbarkeitsberechnung gesucht. Damit sollte es möglich sein, die Stoffabnahme (selten auch eine "scheinbare Zunahme" wie die Aktivierung von Enzy. men usw.) zu umschreiben und aufgrund der experimentell bestimmten Kenngrößen (Geschwindigkeitskonstanten $\lambda$, deren Standardabweichung $\sigma_{\lambda}$, Gesamtanalysenfehler $\sigma_{\text {oij }}$ ) sowie durch Vorgabe der Verlusttoleranz und der Ausschußwahrscheinlichkeit eine Mindesthaltbarkeit zu prognostizieren.

\section{Zerfallsgesetz}

Bei der Haltbarkeitsprüfung werden in einem ersten Schritt die durch Zersetzungsreaktionen hervorgerufenen stofflichen Änderungen bei vorgewählten Lagerungsbedingungen experimentell erfaßt. Aus den Stoffmen-
gen-Zeit- bzw. Konzentration-Zeit-Relationen läßt sich die Reaktionsordnung (2)

- durch die Halbwertszeit-Methode;

- nach der Powell schen Methode (mit dimensionslosen Parametern);

- nach der van't Hoff schen Methode (Messung der Anfangsgeschwindigkeit für verschiedene Ausgangskonzentrationen);

- direkt aus den Geschwindigkeitsdaten

ermitteln.

Wie wir feststellen konnten, gelingt es oft, die Konzentrationsabnahme $c_{(t)}$ eines Wirkstoffes (über die Halbwertszeitkonzentration hinaus) durch eine Bruttoreaktion 1. Ordnung

$$
c_{(t)}=c_{0} e^{-\lambda t}
$$

wiederzugeben (3) (cf. hierzu die Anwendungsbeispiele).

Zudem laufen die Zersetzungsreaktionen bei den zur Haltbarkeitsprüfung anstehenden Produkten meist langsam ab und erreichen im beobachteten Zeitraum selten eine Abnahme von $>20 \%$. Damit läßt sich auch bei komplexen Reaktionssystemen (Folgereaktionen u.a.) die Stoffänderung durch Gl. (1) genügend genau erfassen. Die weiteren Betrachtungen basieren aus diesen Gründen auf der Gültigkeit einer Reaktion 1. Ordnung. Für die seltenen Fälle wo dies nicht zutrifft, kann unser Haltbarkeitsberechnungsmodell leicht angepaßt werden. 


\section{Definitionen}

\section{Allgemein}

Die Deklaration $\mathrm{c}_{\mathrm{o}}$ entspricht dem Mittelwert der Konzentration eines Wirkstoffes zum Zeitpunkt $t_{o}$ (bei Kontrollproben würde dies dem Sollwert eines bestimmten Bestandteils entsprechen).

Als Verlusttoleranz $\alpha$ bezeichnen wir den Anteil, um welchen die Konzentration des Wirkstoffes höchstens abnahmen darf. Das Festlegen der Verlusttoleranz obliegt dem Hersteller, sofern nicht vom Gesetzgeber entsprechende Verordnungen vorliegen.

Die prognostizierte Haltbarkeit entspricht der berechneten Verfallzeit $t_{p}$, in welcher ein Anteil $\beta$ (Ausschußwahrscheinlichkeit) von Meßwerten für die Konzentration der instabilsten Komponente unter den Grenzwert $c_{m}$ fällt. Es gilt definitionsgemäß:

$$
c_{m}=(1-\alpha) c_{0}
$$

\section{Meßwerte, abgeleitete und statistische Größen}

$t_{k}$ : Zeit der k-ten Messung; $k=0 \ldots \infty$

$c_{\mathrm{kij}}$ : Konzentration der Messung $\mathrm{j} ; \mathrm{j}=1,2 \ldots \mathrm{m}$ der Stichprobe $\mathrm{i} ; \mathrm{i}=1,2 \ldots \mathrm{n}$, zur Zeit $\mathrm{t}_{\mathrm{k}}$

$\bar{c}_{k i}$ : Mittelwert der gemessenen Konzentrationen der Stichprobe i zur Zeit $t_{k}$

$c_{\mathbf{k i}}$ : wahrer Wert von $\bar{c}_{\mathbf{k i}}$

$\bar{c}_{k}$ : Mittelwert der gemessenen Konzentrationen aller Stichproben zur Zeit $\mathbf{t}_{\mathbf{k}}$

$c_{k}$ : wahrer Wert von $\bar{c}_{k}$

$\sigma: \quad$ wahre Standardabweichung

$\sigma_{\mathrm{kij}}$ : Standardabweichung der Einzelmessungen $c_{\mathrm{kij}}$ um $c_{k}$

$\sigma_{\mathbf{k i}}:$ Standardabweichung des Mittelwerts $\bar{c}_{\mathbf{k} \mathbf{i}}$ um $c_{\mathbf{k}}$

$\sigma_{\mathrm{a}}$ : Standardabweichung der Analyse

$\sigma_{\text {oij }}$ : Gesamtanalysenfehler (d.h. Standardabweichung der Chargenvariabilität und Analyse)

s: beste Schätzung der Standardabweichung

$\kappa$ : $\quad$ einseitige Signifikanzschranke der standardisierten Normalverteilung für $\beta$ (vgl. 1.c. (4)).

\section{Mathematische Abkürzungen}

Die Mittelwertbildung wird folgendermaßen vereinfacht dargestellt:

$$
i, j \overline{c_{k i j}}=\frac{1}{n}\left[\sum_{i=1}^{n} \frac{1}{m}\left(\sum_{j=1}^{m} c_{k i j}\right)\right] .
$$

Für die Aussage, daß $\bar{c}_{\mathbf{k i}}$ der Schätzwert von $c_{\mathbf{k}}$ ist, gilt die Darstellung $c_{\mathbf{k i}} \Leftarrow \bar{c}_{\mathbf{k i}}$.

\section{Annahmen über die Verteilungsfunktionen}

Wie eingehende Vorversuche gezeigt haben, dürfen unter Berücksichtigung der in der Einleitung und unter Anwendungsbeispiele getroffenen Fèststellungen folgende Annahmen getroffen werden:

a) Normalverteilung der Wirkstoffanfangskonzentrationen $c_{o i}$ in der Stichprobe i um den Mittelwert $c_{o}$ (Deklaration) mit der Standardabweichung $\sigma_{0}$.

b) Normalverteilung der relativen Analysenfehler $\delta_{\mathrm{a}}$ um den Mittelwert 0 mit der Standardabweichung $\sigma_{\mathrm{a}}$.

c) Normalverteilung der Geschwindigkeitskonstanten $\lambda_{i}$ in der Stichprobe i um den Mittelwert $\lambda$ mit der Standardabweichung $\sigma_{\lambda}$.

d) Die Standardabweichungen $\sigma_{o}$ und $\sigma_{\mathrm{a}}$ sowie $\sigma_{\lambda}$ sind statistisch unabhängig.

e) Änderung der Wirkstoffkonzentration mit der Zeit gemäß Gl. (1).

\section{Berechnung der Haltbarkeit}

Die zur Lösung wichtigen Zusammenhänge sind in Abbildung 1 dargestellt.

Zum Zeitpunkt $t_{0}$ (wird im folgenden immer Null gesetzt) sind die wahren Konzentrationen der Stichproben um den Mittelwert. $c_{o}$ der Charge verteilt. Die Konzentrationen der Stichproben und damit der Mittelwert nehmen mit der Zeit ab. Würden im Zeitpunkt $t_{p}$ Messungen durchgeführt, so wären die Meßwerte um $c_{p}$ mit der Standardabweichung $\sigma_{\text {pij }}$ näherungsweise normalverteilt (vgl. Anhang). $\sigma_{\text {pij }}$ hängt von den Standardabweichungen der Stichproben, der Geschwindigkeitskonstanten und den Meßfehlern ab. Ein Anteil $\beta$ von Meßwerten liegt dann unterhalb $\left(c_{p}-\kappa \sigma_{\mathrm{pij}}\right)$. Setzt man diesen Wert gleich dem Grenzwert $c_{m}$, so folgt

$$
c_{p}-\kappa \sigma_{p i j}=c_{m}=(1-\alpha) c_{o} \text {. }
$$

Auf die umfangreiche Herleitung der exakten Lösung wird hier verzichtet. Die Berechnungen führen zu Glei-

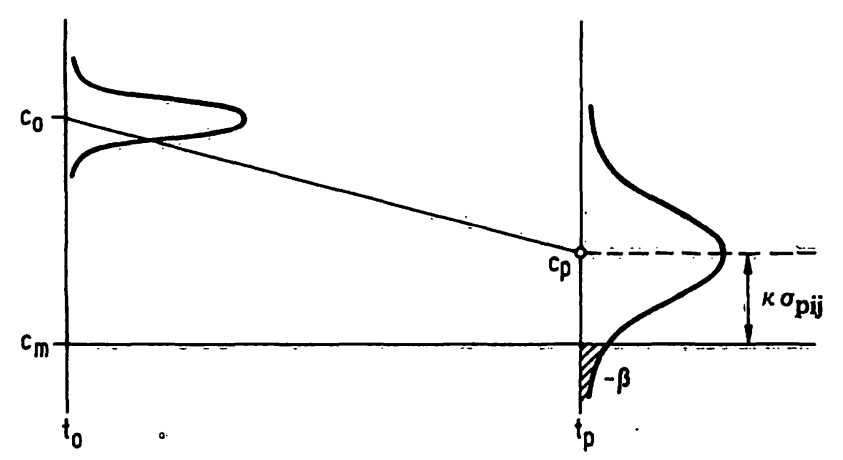

Abb. 1. Verteilungsfunktion der wahren Konzentrationen zum Zeitpunkt $t_{o}=0\left(c_{o}\right)$ und diejenige der Messungen zum Zeitpunkt $t_{p}$ (Mittelwert $c_{p}$ mit Standardabweichung $\left.\sigma_{\mathrm{pij}}\right)$. 
chungen mit Fehlerintegralen, die nur mit einem Digitalrechner gelöst werden können.

Vom Labor aus besteht selten ein direkter Zugriff zum Computer, und Tabellenwerke sind umständlich zu handhaben. Deshalb haben wir nach einer Näherungslösung für $t_{p}$ gesucht, die sich bequem mit den üblichen, programmierbaren Kleinrechnern berechnen läßt.

Nach Einsetzen der Näherungsgleichungen für

$$
c_{p}=c_{o} e^{-\lambda t} p
$$

und

$$
\sigma_{\mathrm{pij}}^{2}=c_{\mathrm{p}}^{2}\left(\left(\frac{\sigma_{\text {oij }}}{c_{\mathrm{o}}}\right)^{2}+\left(\frac{\sigma_{\lambda}}{\lambda}\right)^{2}\left(\lambda t_{\mathrm{p}}\right)^{2}\right)
$$

(vgl. Anhang Gl. (4a) und Gl. (9a) in Gl. (3), sowie Ordnen ergibt sich:

$$
\lambda t_{p}=\ln \frac{1-\kappa \sqrt{\left(\frac{\sigma_{\text {oij }}}{c_{o}}\right)^{2}+\left(\frac{\sigma_{\lambda}}{\lambda}\right)^{2}\left(\lambda t_{p}\right)^{2}}}{1-\alpha} .
$$

Für $\sigma_{\lambda}=0$ wird damit eine explizite Lösung für die Verfallzeit $t_{p}$ erhalten. Im allgemeinen Fall $\sigma_{\lambda}>0$ läßt sich $t_{p}$ durch ein Iterationsverfahren einfach berechnen. Bei allen sinnvollen Anwendungen weicht $\alpha$ wenig von $0 \mathrm{ab}$ (gleichbedeutend mit $\lambda t_{p} \ll 1$ ).

Die Resultate für viele Parameterkombinationen wurden mit den Ergebnissen eines Computerprogrammes für die exakte Lösung verglichen und Übereinstimmung auf mindestens drei signifikante Ziffern festgestellt.

\section{Bestimmung von $c_{o}$ und $s_{o}$ der Charge}

Aus den Meßwerten $c_{o i j}$ von.mehreren Messungen $j$ an mehreren Stichproben $i$ einer Charge zur Zeit $t_{0}$, können $c_{o}$ und $\sigma_{o}$ bzw. $s_{o}$ wie folgt bestimmt werden:

$$
\begin{aligned}
c_{o i} & \Leftarrow \bar{c}_{o i}=j \overline{c_{o i j}} \\
c_{o} & \Leftarrow \bar{c}_{o}=i \overline{c_{o i}} \\
\sigma_{o}^{2} & \Leftarrow s_{o}^{2}=i \frac{i}{\left(c_{o i}-c_{o}\right)^{2}}
\end{aligned}
$$

wobei es ẑu beāchten gilt:

$$
\begin{aligned}
& \sigma_{\mathrm{a}}^{2}={ }^{i, j} \frac{\left(\frac{c_{\mathrm{ojj}}-c_{o i}}{c_{o i}}\right)^{2}}{c} \\
& \ddot{\sigma}_{o i j}^{2}=\stackrel{i, j}{\left(c_{o i j}-c_{o}\right)^{2}}=\sigma_{o i}^{2}+\left(c_{o} \cdot \sigma_{a}\right)^{2} .
\end{aligned}
$$

\section{Bestimmung von $\lambda$ und $s_{\lambda}$ des Wirkstoffes}

Aus den Meßwerten $c_{k i j}$ von mehreren Messungen $j$ an mehreren Stichproben $i$, beị den Z̈eitpunkten $t_{k}$, können $\lambda$ und $\sigma_{\lambda}$ bzw. $s_{\lambda}$ wie folgt bestimmt werden:

$$
\begin{aligned}
\bar{c}_{k i} & =j \overline{c_{k i j}} \\
\lambda_{i} & =\operatorname{REGR}\left[\ln \left(c_{k i}\right), t_{k}\right]
\end{aligned}
$$

$$
\begin{aligned}
\lambda & \Leftarrow \bar{\lambda}=i \overline{\lambda_{i}} \\
\sigma_{\lambda} & \Leftarrow s_{\lambda}^{2}=i \overline{\left(\lambda_{i}-\lambda\right)^{2}} .
\end{aligned}
$$

REGR: Orthogonale lineare Regression

\section{Rechenprogramm}

Zur Berechnung der Verfallzeit $t_{p}$ nach Gleichung (6) wurde ein Rechenprogramm für die Kleinrechner $\mathrm{HP}-67 / 97^{1}$ ) geschrieben. Dabei wurde berücksichtigt, daß in seltenen Fällen während der Lagerdauer eine „scheinbare Gehaltszunahme" der Wirkstoffe (z.B. Aktivierung der alkalischen Phosphatase EC 3.1.3.1) eintreten kann. In diesem Fall sind $\alpha$ und $\lambda$ negativ einzugeben. Das Rechenprogramm enthält neben der Iteration einen von uns neu entwickelten, konvergenzbeschleunigenden Schritt über eine geometrische Reihe.

\section{Anwendungsbeispiele}

Als Beispiele zur Berechnung der Haltbarkeit werden die Kreatinkinase (EC 2.7.3.2) und saure Phosphatase (EC 3.1.3.2) als kritische Bestandteile einer Kontrollprobe herangezogen. Damit das Datenmaterial nicht zu umfangreich wird, sind nur die Meßresultate einer Charge angegeben, obgleich mehrere Chargen überprüft worden sind.

\section{Analysenmethoden}

Kreatinkinase wurde nach der optimierten Standardmethode der Deutschen Gesellschaft für Klinische Chemie $(5,6)$ (mit N-Acetylcystein als Aktivator) auf dem ABA-100 ${ }^{2}$ ) mit Automatenpackung Boehringer Mannheim, Bestell-Nr. 126357 und entsprechender Arbeitsanleitung bestimmt.

Saure Phosphatase wurde mit Thymolphthaleinmonophosphat als Substrat auf dem Greiner Selective Analyzer GSA II ${ }^{3}$ ) nach Arbeitsanleitung C SP1 (7) bestimmt.

\section{Bestimmung von $\lambda, \sigma_{\lambda}$}

Dazu wurden für jede Meßtemperatur $\left(25^{\circ} \mathrm{C}, 30^{\circ} \mathrm{C}\right.$, $\left.37^{\circ} \mathrm{C}\right)$ je 10 Fläschchen einer lyophilisierten Kontrollprobe, mit bidest. Wasser nach Vorschrift aufgelöst, verwendet.

\section{Bestimmung von $\sigma_{\mathrm{oij}}$}

Von derselben Kontrollprobe wurden zusätzlich 10 Fläschchen eingesetzt.

1) Hersteller: Hewlett-Packard S.A., CH-1217 Meyrin. Die Programmbeschreibung kann bei den Autoren angefordert werden.

2) Hersteller: Abbott Laboratories, Diagnostic Division, Pasadena, CA 91030 , USA.

3) Hersteller: Greiner Electronics AG, CH-4900 Langenthal. 
Die Analysenergebnisse sind in den Abbildungen 2 und 3 sowie in der Tabelle 1 enthalten.

Besser gesicherte Werte für $\lambda$ zur Berechnung der Haltbarkeit können in diesem Fall aus den Arrhenius-Plots durch Regressionsrechnung erhalten werden.

Wie stark $\sigma_{o i j}$ die Verfallzeit $t_{p}$ beeinflußt, sei an einem vorgegebenen Beispiel in Tabelle 2 veranschaulicht.

Man muß darauf achten, daß die Meßfehler möglichst klein bleiben, da sonst die prognostizierte Haltbarkeit durch die statistisch schlecht gesicherten Parameter stark herabgesetzt wird. Dies läßt sich durch die mechanisierte Durchführung spezifischer Analysenmethoden (möglichst zur Erfassung des intakten Wirkstoffes und Mehrfachbestimmungen) erreichen. Während der Stabilitätsprüfung

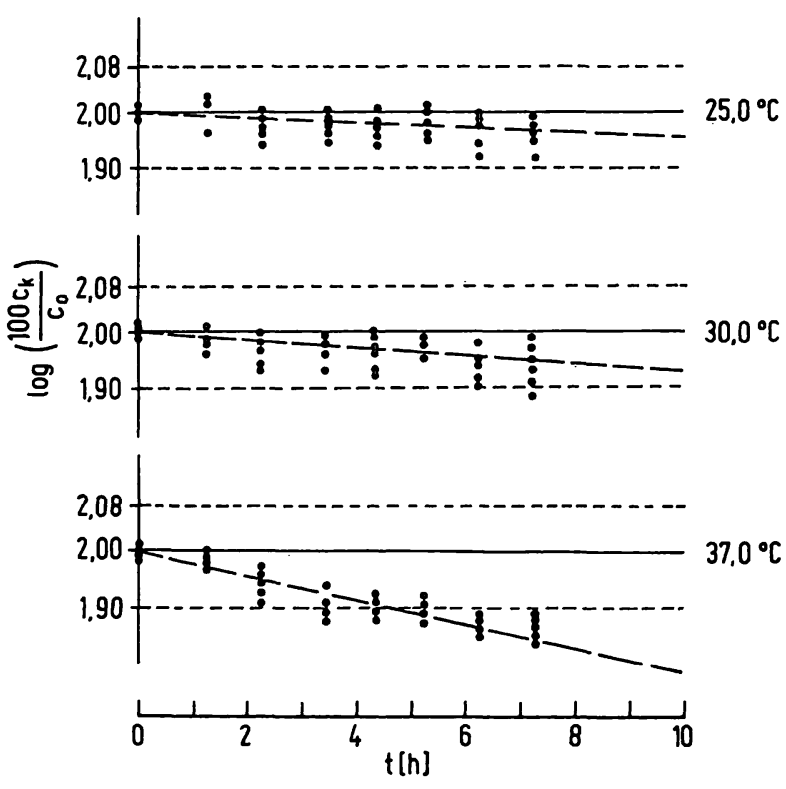

Abb. 2. Konzentration-Zeit-Diagramm: Kreatinkinase als Bestandteil einer Kontrollprobe.
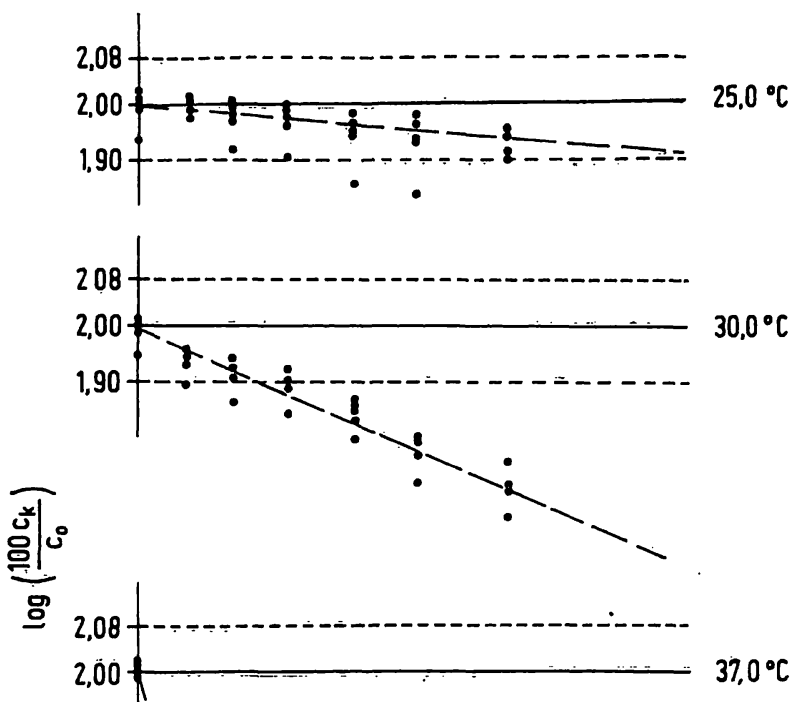

Abb. 3. Konzentration-Zeit-Diagramm: Saure Phosphatase als Bestandteil einer Kontrollprobe.

ist die einmal gewählte Methode unbedingt beizubehalten.

Die prognostizierte Haltbarkeit kann nach Ablauf durch Kontrollmessungen (vgl. hieržu l.c. (8)) überprüft werden, wobei höchstens ein Bruchteil $\beta$ einen Verlust von mehr als $\alpha$ haben darf.

Tab. 1. Aus den Einzelergebnissen berechnete Gesamtanalysenfehler $\sigma_{o j} / c_{o}$, Geschwindigkeitskonstanten $\lambda$ und deren Standardabweichung $\sigma_{\lambda}$ sowie die daraus resultierenden Haltbarkeiten $t_{p}$ für

a) $\lambda$ einzeln bestimmt (Abb. $2 \& 3$ ),

b) $\lambda$ nach Ausgleichsrechnung bestimmt (Abb. 4 \& 5);

Verlusttoleranz $\alpha=0,2$ und Ausschußwahrscheinlichkeit $\beta=0,05$.

\begin{tabular}{|c|c|c|c|c|c|c|c|}
\hline Bestandteil & $\begin{array}{l}\text { Meß- } \\
\text { temperatur } \\
\left({ }^{\circ} \mathrm{C}\right)\end{array}$ & $\begin{array}{l}\sigma_{\mathrm{oij}} / c_{\mathrm{o}} \\
(\%)\end{array}$ & $\begin{array}{c}\sigma_{\lambda} \\
\cdots \\
\left(h^{-1}\right)\end{array}$ & $\begin{array}{l}\lambda \\
\left(h^{-1}\right)\end{array}$ & $\begin{array}{l}t_{p} \\
(h)\end{array}$ & $\begin{array}{l}\lambda \\
\left(h^{-1}\right)\end{array}$ & $\begin{array}{l}t_{p} \\
(h)\end{array}$ \\
\hline Kreatinkinase & $\begin{array}{l}25,0 \\
30,0 \\
37,0\end{array}$ & $\begin{array}{l}4,2 \\
5,3 \\
3,3\end{array}$ & $\begin{array}{l}0,00235 \\
0,00346 \\
0,00239\end{array}$ & $\begin{array}{l}0,00992 \\
0,01790 \\
0,04945\end{array}$ & $\begin{array}{r}13,4 \\
6,9 \\
3,4\end{array}$ & $\begin{array}{l}0,00954 \\
0,01914 \\
0,04803\end{array}$ & $\begin{array}{r}13,8 \\
6,4 \\
3,4\end{array}$ \\
\hline Saure Phosphatase & $\begin{array}{l}25,0 \\
30,0 \\
37,0\end{array}$ & $\begin{array}{l}3,0 \\
2,5 \\
1,5\end{array}$ & $\begin{array}{l}0,00438 \\
0,02356 \\
0,03639\end{array}$ & $\begin{array}{l}0,01895 \\
0,11192 \\
0,69704\end{array}$ & $\begin{array}{l}7,7 \\
1,4 \\
0,3\end{array}$ & $\begin{array}{l}0,02061 \\
0,09654 \\
0,74284\end{array}$ & $\begin{array}{l}7,2 \\
1,5 \\
0,3\end{array}$ \\
\hline
\end{tabular}




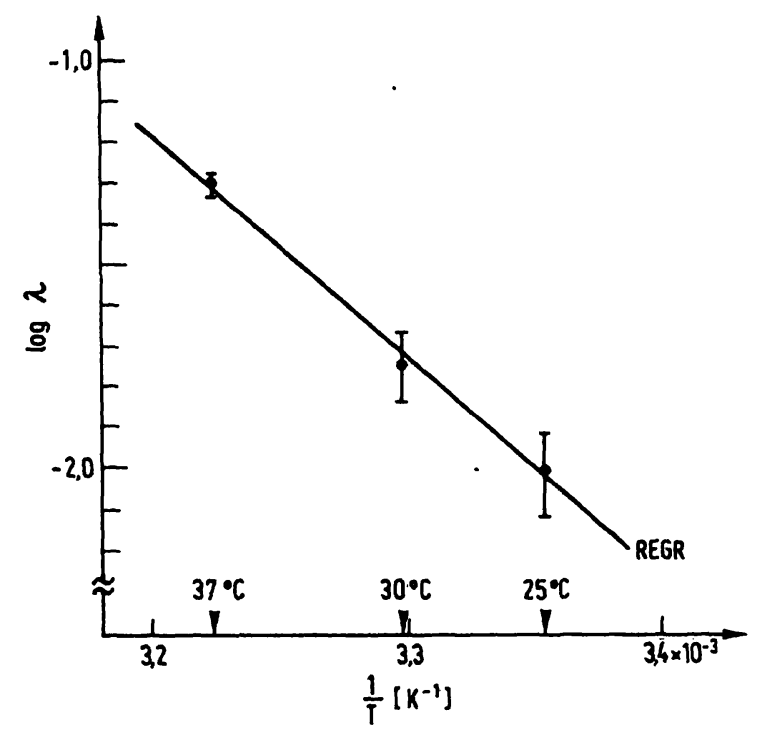

Abb. 4. Arrhenius-Plot: Kreatinkinase als Bestandteil einer Kontrollprobe. Mittelwert $\log \lambda \pm 1$ angegeben.

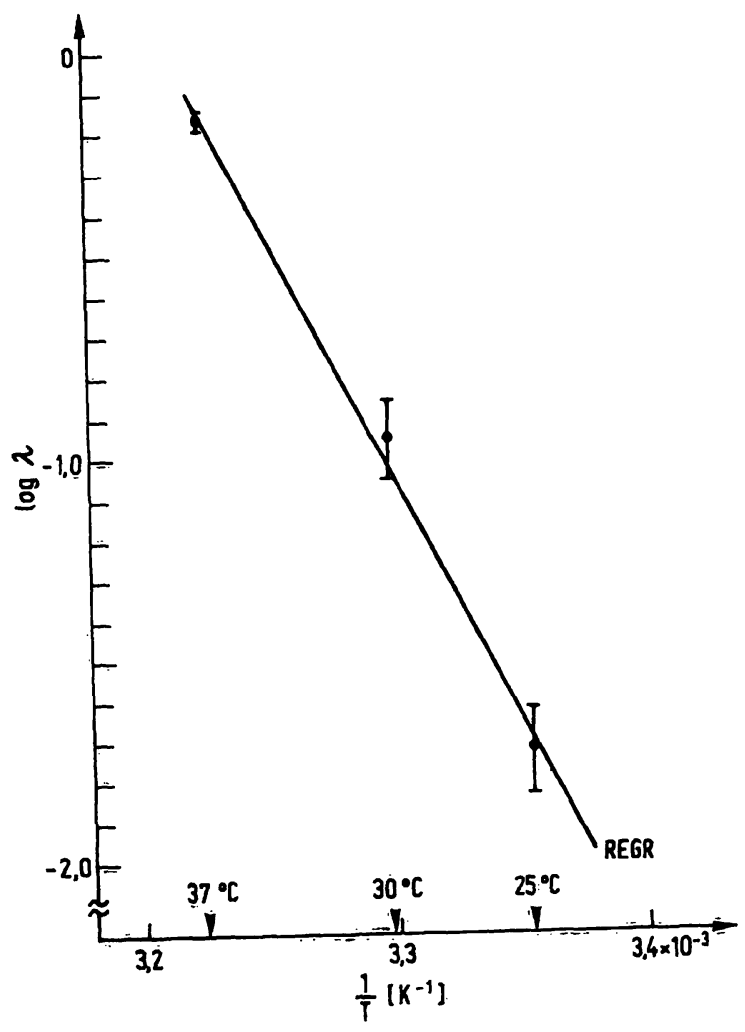

Abb. 5. Arhenius-Plot: Saure Phosphatase als Bestandteil einer Kontrollprobe. Mittelwert log $\lambda \pm$ is angegeben.
Tab. 2. Einfluß der Standardabweichungen $\sigma_{\lambda}$ und $\sigma_{o i j} / c_{o}$ auf die Haltbarkeit.

Anfangsbedingungen:

Geschwindigkeitskonstante $\lambda=10^{-4} \mathrm{~h}^{-1}$; Verlusttoleranz $\alpha$ und Ausschußwahrscheinlichkeit $\beta=0,05$.

\begin{tabular}{|c|c|c|c|c|c|c|}
\hline $\begin{array}{l}\sigma_{\lambda} \\
\left(h^{-1}\right)\end{array}$ & $\begin{array}{c}\sigma_{o i j} / c_{o} \\
(\%)\end{array}$ & 0 & 1 & 2 & 3 & 4 \\
\hline $\begin{array}{l}0 \\
10^{-5} \\
2 \cdot 10^{-5} \\
3 \cdot 10^{-5} \\
4 \cdot 10^{-5} \\
5 \cdot 10^{-5} \\
6 \cdot 10^{-5}\end{array}$ & & $\begin{array}{l}512,9 \\
440,3 \\
385,4 \\
342,5 \\
308,1 \\
280,0 \\
256,5\end{array}$ & $\begin{array}{l}347,1 \\
338,0 \\
317,1 \\
293,0 \\
270,0 \\
249,3 \\
231,0\end{array}$ & $\begin{array}{l}178,4 \\
177,2 \\
173,6 \\
168,3 \\
162,0 \\
155,0 \\
148,0\end{array}$ & $\begin{array}{l}6,90 \\
6,90 \\
6,90 \\
6,89 \\
6,88 \\
6,87 \\
6,85\end{array}$ & $\begin{array}{l}- \\
- \\
- \\
- \\
-\end{array}$ \\
\hline
\end{tabular}

\section{Anhang}

\section{Näherungslösung für $c_{p}$}

Für die Konzentrationen einer Stichprobe i zum Zeitpunkt $t_{p}$ erhält man mit den Abweichungen:

$$
\begin{array}{rlrl}
\epsilon_{0 i} & =c_{o i}-c_{o} & & \text { Gl. (1aa) } \\
\epsilon_{\lambda i} & =\lambda_{i}-\lambda & & \text { Gl. (lab) } \\
\delta_{a i j} & =\left(c_{o i j}-c_{o i}\right) / c_{o i} & \text { Gl. (lac) }
\end{array}
$$

als Meßwert

$$
c_{p i j}=\left(c_{o}+\epsilon_{o i}\right)\left(1+\delta_{a i j}\right) e^{-\left(\lambda+\epsilon_{\lambda i}\right) t_{p}} .
$$

Durch Entwicklung nach $\epsilon_{\mathrm{oi}}, \delta_{\text {aij }}$ und $\epsilon_{\lambda \mathrm{i}}$ und Abbruch nach den Gliedern 1. Ordnung ergibt sich:

$$
c_{p i j}=c_{o} e^{-\lambda t_{p}}\left(1+\frac{\epsilon_{o j}}{c_{o}}+\delta_{a i j}-\epsilon_{\lambda i} t_{p}\right) .
$$

Durch Mittelwertbildung über $j$ und i folgt für die Abnahme des Mittelwertes:

$$
c_{p}=c_{o} e^{-\lambda t_{p}} \text {. }
$$

Näherungslösung für $\sigma_{\mathrm{p} i \mathrm{j}}$

Für die Standardabweichung $\sigma_{\mathrm{pij}}$ der Meßwerte zur Zeit $t_{\mathrm{p}}$ gilt:

$$
\sigma_{\mathrm{pij}}^{2}={ }^{\mathrm{i}, \mathrm{j}} \overline{\left(c_{\mathrm{pij}}-c_{\mathrm{p}}\right)^{2}}
$$

mit

$$
c_{p i j}-c_{p}=c_{p}\left(\frac{\epsilon_{0 i}}{c_{o}}+\delta_{a i j}-\epsilon_{\lambda i} t_{p}\right)
$$

Aus Gleichung (5a) und (6a) folgt

$$
\sigma_{\mathrm{pij}}^{2}=c_{\mathrm{p}}^{2}\left(\left(\frac{\sigma_{\mathrm{o}}}{c_{\mathrm{o}}}\right)^{2}+\sigma_{\mathrm{a}}^{2}+\left(\frac{\sigma_{\lambda}}{\lambda}\right)^{2}\left(\lambda \mathrm{t}_{\mathrm{p}}\right)^{2}\right)
$$

und analog für die Zeit $t_{0}$

$$
\sigma_{o i j}^{2}=\sigma_{0}^{2}+c_{0}^{2} \sigma_{a}^{2} \text {. }
$$

Damit läßt sich Gleichung (7a) weiter vereinfachen zu

$$
\sigma_{\mathrm{pij}}^{2}=c_{\mathrm{p}}^{2}\left(\left(\frac{\sigma_{\mathrm{oij}}}{c_{0}}\right)^{2}+\left(\frac{\sigma \lambda}{\lambda}\right)^{2}\left(\lambda t_{\mathrm{p}}\right)^{2}\right) .
$$




\section{Literatur}

1. Grimm, W. (1973), Pharm. Ind. 35, 79-85.

2. Frost, A. A. \& Pearson, R. G. (1973), Kinetik und Mechanismen homogener chemischer Reaktionen, 2. Aufl., Kap. 2 \& 3, Verlag Chemie, Weinheim.

3. Grimm. W. (1974), Pharm. Ind. 36. 437-442.

4. Wissenschaftliche Tabellen Geigy (1980), Teilband Statistik, 8. Auflage, S. 26, Ciba-Geigy AG, Basel.
5. Empfehlungen der deutschen Gesellschaft für klinische Cliemie: (1977), diese Z. 15, 249-254.

6. Gruber, W. (1978), Clin. Chem. 24, 177-17.8.

7. Methods Handbook (1976), Greiner Electronics Selective Analyzer GSA II.

8. Thiers, R. E., Wu. G. T., Reed. A. H. \& Oliver, L. K. (1976), Clin. Chem. 22, 176-183.

Dipl. Chem. P. Schläptèr und Dr. IF. Hänni F. Hoffimann-La Roche \& Co. AG CH-4002 Bascl 